\title{
Effects of surface charges of gold nanoclusters on long-term in vivo biodistribution, toxicity, and cancer radiation therapy
}

This article was published in the following Dove Press journal:

International Journal of Nanomedicine

27 July 2016

Number of times this article has been viewed

\author{
Jun-Ying Wang' \\ Jie Chen' \\ Jiang Yang ${ }^{2}$ \\ Hao Wang' \\ Xiu Shen' \\ Yuan-Ming Sun' \\ Meili Guo ${ }^{3}$ \\ Xiao-Dong Zhang ${ }^{4}$ \\ 'Tianjin Key Laboratory of \\ Radiation Medicine and Molecular \\ Nuclear Medicine, Institute of \\ Radiation Medicine, Chinese Academy \\ of Medical Sciences and Peking Union \\ Medical College, Tianjin, ${ }^{2}$ Environment, \\ Energy and Natural Resources Center, \\ Department of Environmental Science \\ and Engineering, Fudan University, \\ Shanghai, ${ }^{3}$ Department of Physics, \\ School of Science, Tianjin Chengjian \\ University, ${ }^{4}$ Department of Physics, \\ School of Science, Tianjin University, \\ Tianjin, People's Republic of China
}

Correspondence: Xiao-Dong Zhang Department of Physics, School of Science, Tianjin University, 135 Yaguan Road, Tianjin 300350, People's Republic of China

Tel/fax +86228568 304l

Email xiaodongzhang@tju.edu.cn

Yuan-Ming Sun

Tianjin Key Laboratory of Radiation

Medicine and Molecular Nuclear

Medicine, Institute of Radiation Medicine,

Chinese Academy of Medical Sciences

and Peking Union Medical College,

No 238, Baidi Road, Tianjin 300350,

People's Republic of China

Email sunyuanming@irm-cams.ac.cn

\begin{abstract}
Gold nanoclusters (Au NCs) have exhibited great advantages in medical diagnostics and therapies due to their efficient renal clearance and high tumor uptake. The in vivo effects of the surface chemistry of Au NCs are important for the development of both nanobiological interfaces and potential clinical contrast reagents, but these properties are yet to be fully investigated. In this study, we prepared glutathione-protected Au NCs of a similar hydrodynamic size but with three different surface charges: positive, negative, and neutral. Their in vivo biodistribution, excretion, and toxicity were investigated over a 90-day period, and tumor uptake and potential application to radiation therapy were also evaluated. The results showed that the surface charge greatly influenced pharmacokinetics, particularly renal excretion and accumulation in kidney, liver, spleen, and testis. Negatively charged Au NCs displayed lower excretion and increased tumor uptake, indicating a potential for NC-based therapeutics, whereas positively charged clusters caused transient side effects on the peripheral blood system.
\end{abstract}

Keywords: gold clusters, in vivo toxicity, long-term, cancer therapy

\section{Introduction}

Gold nanoclusters (Au NCs) are well established in medicine due to their excellent biological properties that include excretion in the form of small molecules and ultrahigh tumor uptake. ${ }^{1-3}$ Unlike traditional large size nanoparticles (NPs, $\left.>10 \mathrm{~nm}\right),{ }^{4-6}$ typical Au NCs have a renal clearance cutoff of less than $5.5 \mathrm{~nm},{ }^{7}$ to easily escape the reticuloendothelial system (RES) and accumulate in the tumor tissue., ${ }^{3,89}$ Therefore, they are widely applied in imaging, drug deliveries, and cancer radiation therapies. ${ }^{1-3,9-17}$ As a preclinical molecule, potential toxicities could hinder further medical applications. In contrast to small chemical molecules, nanosized particles with larger volumetric specific surface area are more reactive. ${ }^{18,19}$ The dimension, shape, surface charge, and coating can all contribute to the overall toxicity of NPs. ${ }^{20-23}$ To exploit realistic applications of Au NCs, all these factors should be taken into account and studied carefully to ensure the potential side effects on humans are minimized. Various in vitro studies were performed but with conflicting results. Connor et al reported that Au NPs are not inherently toxic to human K562 cells unless they are taken up, ${ }^{24}$ and Shukla et al suggested Au NPs did not possess any immunological or cellular toxicity. ${ }^{25}$ However, other studies indicated that Au NPs could lead to oxidative damage to lung fibroblasts as well as cytotoxicity and genotoxicity to small airway epithelial cells. ${ }^{26,27}$ The reasons for these conflicting results may be attributed to the different surface charges of Au NCs and different cells lines employed in the experiments, which increase uncertainties in relation to toxicities. Furthermore, 
in vitro experiments are very helpful for high-throughput screening of a large number of nanomaterials, but the in vivo biological system is far more complicated and the Au NCs may interact with various proteins and cell types. Our previous work has shown that bovine serum albumin (BSA)- and glutathione (GSH)-protected Au NCs could cause inflammation and kidney damage in the short term, but the toxicity response would be eliminated after 28 days of treatment with $94 \%$ being excreted as GSH-Au NCs. Other reports also indicated good biocompatibility in vivo. ${ }^{28-31}$ However, the effects of surface charge in the physiological environment have not been studied. We therefore investigated biodistribution, excretion, and toxicity in this study and evaluated the biocompatibility of Au NCs with positive, negative, and neutral surface charges.

NPs have highly active surfaces and surface charge is a significant factor in determining their biological characteristics. Indeed, surface charge has a distinct impact on cell toxicity and can vary with cell type. ${ }^{32-34}$ Surface charge can affect adhesion to cell membranes as well as uptake efficiency. ${ }^{35}$ Positively charged NPs bind strongly to serum components in the blood via noncovalent interactions with proteins and electrostatic interactions with the cell surface. ${ }^{36-38}$ Furthermore, these positively charged NPs can be easily taken up by nonphagocytic cells and cause more disruption of the membrane integrity and lysosomal and mitochondrial damage than their negatively charged counterparts. ${ }^{39-42}$ Positively charged Au NPs, therefore, display high uptake in the liver. A better understanding of the effects of surface charge is, therefore, important for both fundamental knowledge regarding potential nano-bio interfaces and medical applications.

To address these concerns of gold clusters, we investigated the long-term in vivo biological performances with different surface charges (positive, negative, and neutral). All three types had a surface coating of GSH and were therefore of similar size. Tumor uptake was also evaluated.

\section{Materials and methods Materials and synthesis}

Orange-emitting Au NCs were synthesized using a previously reported method. ${ }^{43}$ Briefly, freshly prepared aqueous solutions of $\mathrm{HAuCl}_{4}(20 \mathrm{mM}, 0.50 \mathrm{~mL})$ and $\mathrm{GSH}(100 \mathrm{mM}, 0.15 \mathrm{~mL})$ were mixed with $5 \mathrm{~mL}$ of ultrapure water at $25^{\circ} \mathrm{C}$. The reaction mixture was heated to $70^{\circ} \mathrm{C}$ with stirring $(500 \mathrm{rpm})$ for 24 hours. The resultant solution was light yellow under room light. Au NCs were purified using ultrafiltration (with a cutoff of $3 \mathrm{kDa}$ molecular weight). For positively charged Au NCs, $1 \mathrm{mmol} \mathrm{Au} \mathrm{NCs} \mathrm{was} \mathrm{mixed} \mathrm{with} 3 \mathrm{mmol}$ ethylenediamine and stirred at room temperature (RT) for 30 minutes, then
10 mmol 1-(3-dimethyl-amino propyl)-3-ethylcarbodiimide hydrochloride $(\mathrm{EDC} / \mathrm{HCl})$ was added and incubated for 3 hours. Samples were purified using ultrafiltration devices (cutoff weight $3 \mathrm{kDa}$ ). For negatively charged Au NCs, 3 mmol ethanedioic acid and $30 \mathrm{mmol} \mathrm{EDC} / \mathrm{HCl}$ were mixed and stirred for 30 minutes at RT, then $1 \mathrm{mmol}$ Au NCs (synthesized as mentioned previously) was added and incubated for 3 hours at RT. Impurities were removed by ultrafiltration $(3 \mathrm{kDa})$. Raw products and purified Au NCs were stable at $4^{\circ} \mathrm{C}$ for 6 months showing negligible change in their optical properties.

\section{Characterization}

Transmission electron microscopy (TEM) images were obtained using a JEOL JEM-2100F microscope operated at $200 \mathrm{kV}$ (JEOL, Tokyo, Japan). Zeta potential and hydrodynamic diameter data were acquired by using a NanoZS Zeta-sizer particle analyzer (Malvern Instruments, Malvern, UK). Sample solutions were prepared by diluting Au NCs to $0.01 \mathrm{mM}$ with PBS solution (pH 7.4). Data were acquired in the phase analysis light scattering mode at $25^{\circ} \mathrm{C}$.

UV-vis (ultraviolet-visible) absorption spectra were obtained using a Shimadzu UV-1800 spectrophotometer (Shimadzu Inc., Kyoto, Japan). Photoluminescence spectra were recorded by a fluorescence spectrophotometer (F4600 Hitachi; Hitachi Ltd., Tokyo, Japan). The stability of differently charged Au NCs was evaluated by observing changes in fluorescence spectra over time. The Au NCs (3 mM, $0.5 \mathrm{~mL}$ ) were diluted twofold in fetal bovine serum (FBS), and fluorescence spectra were measured at the time points of $0.5,2,6,12$, and 24 hours.

\section{Animal treatments and sample collection}

All animals were purchased, maintained, and handled under protocols approved by the guidelines set by the Institute of Radiation Medicine, Chinese Academy of Medical Science. The experiments also received ethical approval from Institute of Radiation Medicine, Chinese Academy of Medical Sciences Institutional Review Board. One hundred and sixty, 11-week-old male C57 mice were housed in standard 12-hour light/dark cycles with food and water given ad libitum. Mice were randomized into control and treatment groups of 1, 7, 30,60 , and 90 days $(n=8)$. Au NCs and distilled water (control) were intraperitoneally injected at a dose of $5.9 \mathrm{mg} / \mathrm{kg}$. After 1, 7, 30, 60, and 90 days of administration, animals were sacrificed using isoflurane anesthesia and angiocatheter exsanguination with PBS. Blood, liver, kidney, spleen, heart, lung, and testis were collected and weighed for further excretion, biodistribution, and pathological investigations. 


\section{Biodistribution}

Tissue samples from mice treated with $\mathrm{Au}$ NCs $(0.5 \mathrm{~g})$ were digested in $6 \mathrm{~mL}$ nitric acid in a microwave (Mars 5; CEM, Kamp-Lintfort, Germany). Then the samples were condensed to 1-2 mL using an electric heater. The resultant solution was transferred to polyethylene terephthalate bottles and adjusted to $50 \mathrm{~g}$ using $2 \%$ nitric acid. The concentration of Au was determined by inductively coupled plasma mass spectrometry (Agilent, 7500 CE; Agilent Technologies, Santa Clara, CA, USA).

\section{In vivo toxicity}

C57 male mice were divided into 20 groups, including controls, $\mathrm{Au}, \mathrm{Au}+$, and $\mathrm{Au}-$ groups for 1, 7, 30, 60, and 90 days postinjection. Approximately, $0.2 \mathrm{~mL}$ of Au NCs (3 mM) was intraperitoneally administered, and all the mice were weighted every day and checked for behavioral changes. At different time points, mice were sacrificed, and blood samples of the major organs were subjected to toxicity analysis. A standard saphenous blood collection technique was used to draw $1 \mathrm{~mL}$ blood, and $20 \mu \mathrm{L}$ was placed in a potassium ethylenediamine tetraacetic acid collection tube for hematology analysis. Serum was separated by centrifuging the blood at $250 \times g$ for 5 minutes to remove the cellular fraction for biochemistry analysis. Kidneys were preserved, fixed in 10\% neutral buffered formalin, processed into paraffin, and stained with hematoxylin and eosin for pathology analysis using a digital microscope.

\section{In vivo radiotherapy}

All animals were purchased, maintained, and handled under protocols approved by the Institute of Radiation Medicine, Chinese Academy of Medical Science. Tumor models (U14) were produced by the subcutaneous injection of $0.1 \mathrm{~mL} 2 \times 10^{6}$ cells $/ \mathrm{mL}$ in PBS into the right armpit of female BALB/c mice. When tumor volumes reached $100-300 \mathrm{~mm}^{3}$, mice were randomly divided into four groups (control + radiation, neutral
$\mathrm{Au} \mathrm{NCs}+\mathrm{rad}$, positive $\mathrm{Au} \mathrm{NCs}+\mathrm{rad}$, and negative Au NCs $+\mathrm{rad}$ ). Solutions of Au NCs or distilled water (control) were intraperitoneally injected into mice and 30 minutes later they were exposed to 5 Gy gamma rays. ${ }^{137} \mathrm{Cs}$ with an activity of 3,600 $\mathrm{Ci}$ and a photon energy of $662 \mathrm{keV}$ was employed. Tumor size was measured every 2 days and calculated as (tumor length) $\times(\text { tumor width })^{2} / 2$.

\section{Statistical analysis}

Data are presented as mean \pm standard deviation, and independent Student's $t$-test was used for statistical analysis.

\section{Results and discussion Synthesis and characterization of GSH- $\mathrm{Au}_{33} \mathrm{NCs}$}

$\mathrm{Au}$ NCs were synthesized and purified according to a predefined protocol. ${ }^{43}$ Either ethanediamine or ethanedioic acid was conjugated to $\mathrm{GSH}-\mathrm{Au}_{33}$ using $\mathrm{EDC} / \mathrm{HCl}$ as a condensing reagent to generate a positively or negatively charged surface, respectively. A representative TEM image (Figure 1A) revealed that as-synthesized $\mathrm{Au} \mathrm{NCs}$ (neutral) dispersed well in solution and were of a homogeneous dimension $(<5 \mathrm{~nm})$, and a uniform average size of $3 \mathrm{~nm}$ was confirmed by dynamic light scattering (Figure 1B). Negatively and positively charged $\mathrm{Au} \mathrm{NCs}$ had an average size of 3.2 and $3.7 \mathrm{~nm}$, respectively. Differences in the surface charge were analyzed by zeta potential (Figure 1C), and surface potentials of $-0.1 \mathrm{mV},+6.3 \mathrm{mV}$, and $-3.9 \mathrm{mV}$ were obtained for neutral, positively, and negatively charged $\mathrm{Au} \mathrm{NCs}$, respectively. Optical properties were determined by measuring the fluorescence intensity at $610 \mathrm{~nm}$ (Figure 1D).

\section{Blood plasma stability}

$\mathrm{Au}$ NCs interact with various plasma proteins in the blood plasma. Adsorption of plasma proteins on the surface makes

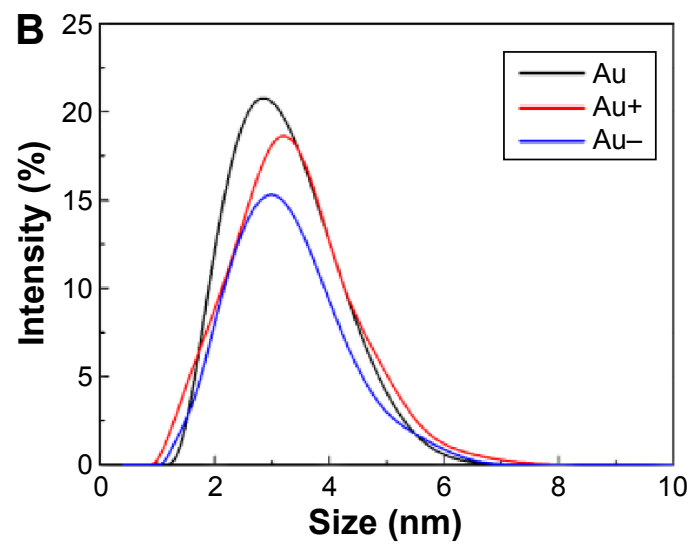

Figure I (Continued) 

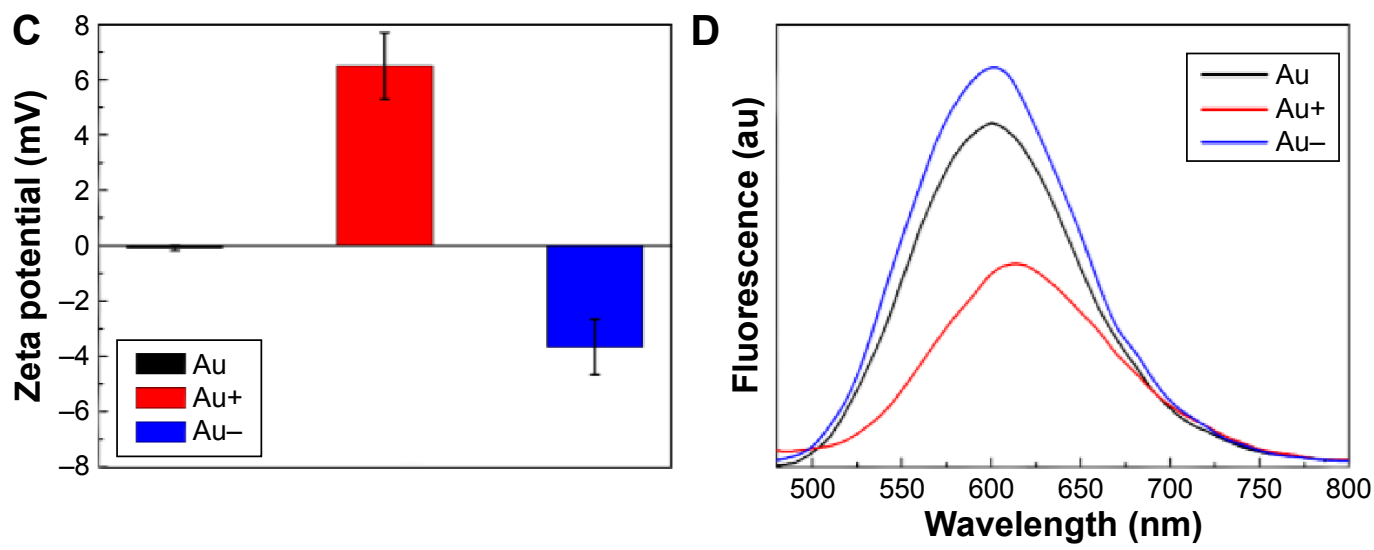

Figure I Physical properties of gold cluster.

Notes: (A) TEM image of neutral Au NCs (scale bar $10 \mathrm{~nm}$ ). (B) Dynamic light scattering of Au NCs with different surface charges. The hydrodynamic diameter is 3, 3.2, and $3.7 \mathrm{~nm}$ for neutral, negative, and positive Au NCs, respectively. (C) Surface charge characterized by zeta potential $(-0.1 \mathrm{mV},-3.9 \mathrm{mV}$, and $+6.3 \mathrm{mV}$ for neutral, negative, and positive Au NCs, respectively). (D) Photoluminescence spectra of Au NCs with different surface charges excited at $365 \mathrm{~nm}$

Abbreviations: TEM, transmission electron microscope; Au NCs, gold nanoclusters; au, arbitrary units.

NCs more easily susceptible to opsonization and prolongs the clearance via blood circulation. Stability in blood plasma is thus important for NCs to exert any potential therapeutic effects. Au NCs were diluted in FBS, and the photoluminescence intensity was measured at different time points within
24 hours. As shown in Figure 2, only a slight decrease in fluorescence intensity occurred within 12 hours, indicating interactions with proteins and formation of "protein corona". However, the fluorescence intensity of all clusters was stabilized to some extent after 12 hours of incubation, and after

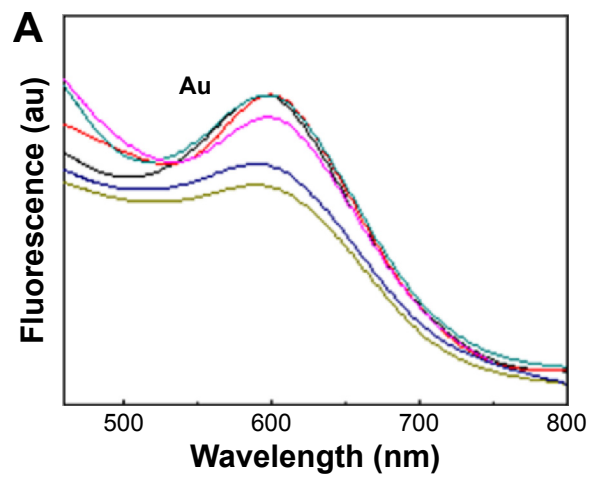

B
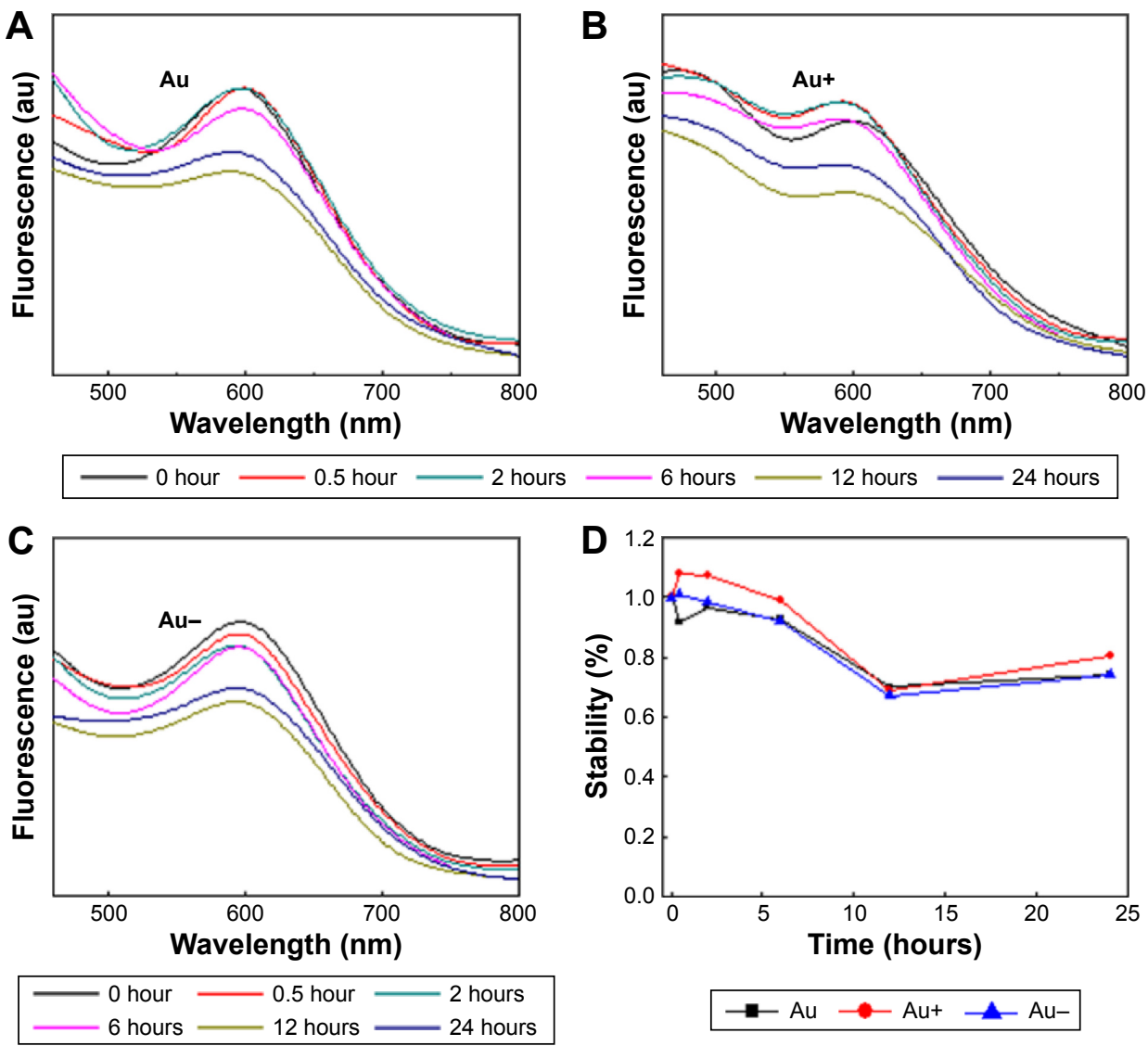

Figure 2 Stability of gold clusters.

Notes: (A-C) Time-dependent UV absorption of Au, Au+, and Au NCs in the blood plasma. (D) Changes of luminescence intensity (\%) in blood plasma over time. Abbreviations: UV, ultraviolet; Au, gold; NCs, nanoclusters; au, arbitrary units. 
24 hours, it decreased to $75 \%$ for neutral and negative $\mathrm{Au}$ NCs and to $80 \%$ for positive Au NCs. It can be observed from Figure $2 \mathrm{~A}-\mathrm{C}$ that the fluorescence intensity of neutral and negative Au NCs continued to decrease over 12 hours without any shift in the center of the main emission peak, whereas there was a slight blue-shift and peak broadening for positive $\mathrm{Au} \mathrm{NCs}$, due to their higher affinity for plasma proteins under physiological conditions. Negatively charged Au NCs bearing acidic groups (dicarboxyl) tended to absorb proteins with a $\mathrm{pI}>5.5$, such as apolipoprotein, whereas positively charged $\mathrm{Au}$ NCs bearing basic groups preferred proteins with a $\mathrm{pI}<5.5$, such as albumin. Au NC-protein complexes are in a dynamic balance and change over time. ${ }^{44-46}$ Therefore, measuring their fluorescence intensity is useful for evaluating their stability in blood plasma.

\section{Biodistribution and excretion}

In vivo biodistribution and excretion profiles are crucial for evaluating biocompatibility, therapeutic potential, and possible toxicity of NCs. Low accumulation in nontargeted organs is desirable, as is faster excretion, especially for NCs of ultrasmall size $(<5 \mathrm{~nm})$. The influence of surface charge on biodistribution and excretion was investigated over a relatively long period of 90 days. As shown in Figure 3, highly vascularized kidneys, testis, liver, and spleen were the most targeted organs. The kidneys retained the largest amount of $\mathrm{Au} \mathrm{NCs,} \mathrm{and} \mathrm{metabolic} \mathrm{clearance} \mathrm{was} \mathrm{clearly} \mathrm{abnormal}$ compared with other organs, with a characteristic biphasic biodistribution as reported by us previously. ${ }^{14}$ All organs except kidneys continued to excrete $\mathrm{Au}$ NCs for 30 days, but the amount of Au NCs refluxed during 30-90 days, as indicated by the $\mathrm{V}$-shaped distribution profiles. Neutral Au NCs were more vulnerable to RES uptake than charged NCs. Target-oriented differences in biodistribution - due to differences in the charges - could be observed, which indicated that positive Au NCs preferred to accumulate in the kidneys. ${ }^{47-49}$ Additionally, higher retention in other RES organs such as liver and spleen at 1 day after administration indicate fast clearance from the bloodstream.

In contrast, negative $\mathrm{Au}$ NCs displayed a higher residual amount in liver $(6,741.1 \mathrm{ng} / \mathrm{g})$ than positive NCs after 90 days, which suggests a longer circulation time in vivo. Based on the amount of Au present in all organs, both positively and negatively charged Au NCs were excreted slightly more slowly than neutral clusters over the 90-day period. Positive Au NCs exhibited the largest overall accumulation in the kidney, spleen, lung, and heart $(21,758,7,559,2,007$, and $2,487 \mathrm{ng} / \mathrm{g}$, respectively) after 90 days, suggesting a wider distribution in vivo. Liver and testis were the main organs targeted by negative Au NCs $(6,741$ and 5,514 ng/g,
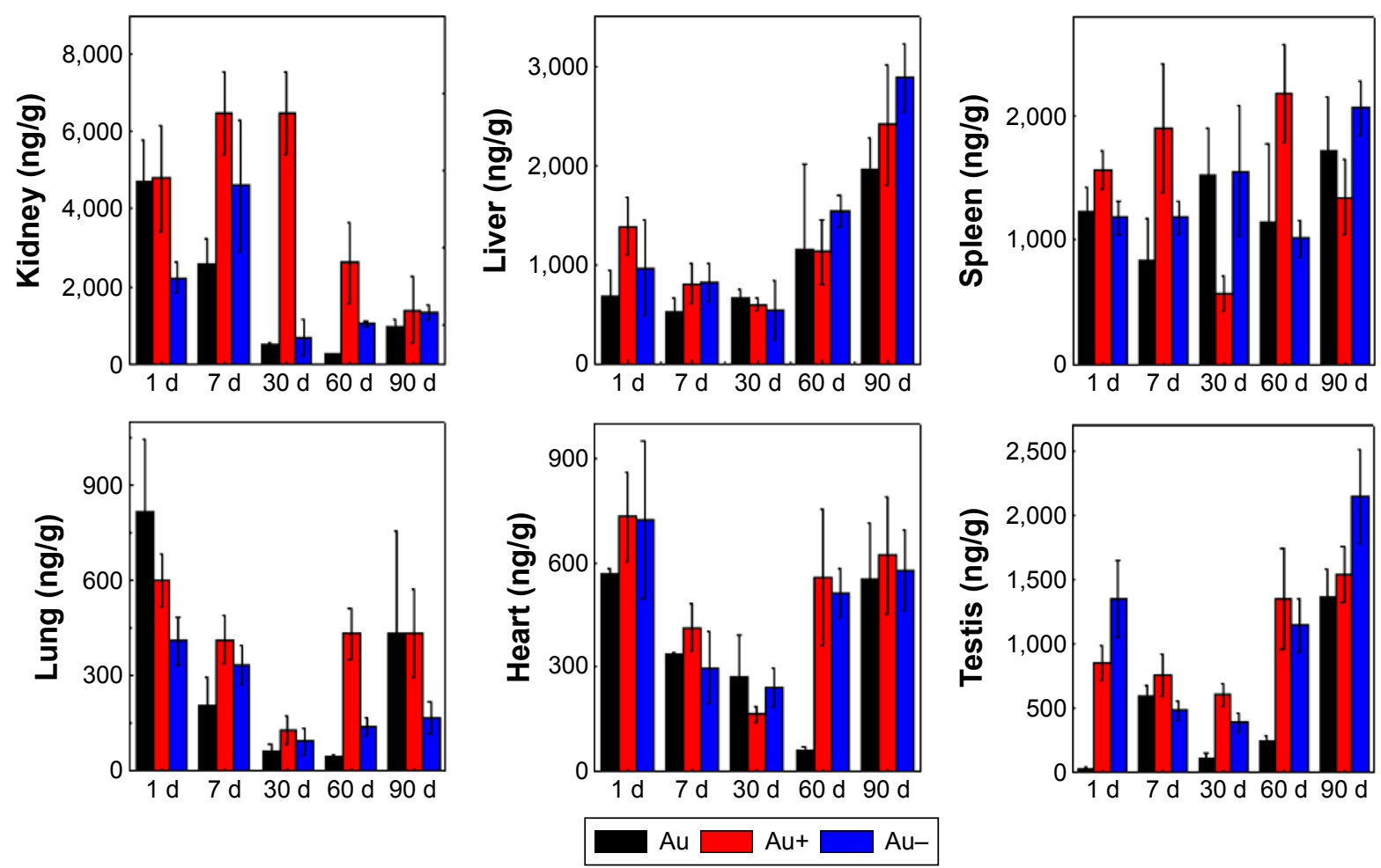

Figure 3 Biodistribution of $\mathrm{Au}, \mathrm{Au+}$, and $\mathrm{Au}-$ in the main organs at I, 7, 30, 60, and 90 days after administration. Abbreviations: Au, gold; d, days. 
respectively) during 90 days of observation. It is well known that $\mathrm{NCs}^{50}$ with high surface activities can be easily opsonized to form aggregates that are readily identified by macrophages and captured by blood-rich organs, of which liver and spleen are the most prominent. A higher accumulation of positive Au NCs in kidneys indicated that this is the main target organ of these clusters. However, relatively higher levels in other organs suggest that excretion of charged Au NCs is slower than neutral Au NCs. The accumulation of neutral Au NCs in kidneys could reflect a faster renal clearance, as was reported by us previously. ${ }^{50}$ The biodistribution of the three different surface-charge variants could reflect typical renal clearance of NCs, while the biphasic distribution suggested that NCs can be easily retained in the tissues or organs through the endothelial leakiness effect. ${ }^{51}$ The target selectivity of $\mathrm{Au}$ NCs with different surface charges is consistent with the in vivo biodistribution of Au composite nanodevices $(5 \mathrm{~nm})$ with different surface charges. ${ }^{47}$

\section{In vivo toxicity}

Determination of the in vivo toxicity of NCs is essential prior to any clinical applications, especially when the synthetic processes vary and diverse surface modifications are incorporated. For a promising candidate of $\mathrm{NCs}$, it is interesting to investigate the influence of surface charges of Au NCs on their toxicities. Au NCs were intraperitoneally injected into C57 mice at a dose of $5.9 \mathrm{mg} / \mathrm{kg}$ and hematology, biochemistry, and pathology tests were performed on days 1, 7, 30, and 90. Different surface charges could cause side effects on the peripheral blood system (Figure 4). Specifically, red blood cells, platelets (PLT), hemoglobin (HGB), mean corpuscular hemoglobin, and mean corpuscular hemoglobin concentration (MCHC) increased dramatically after 7 and 30 days in mice treated with positive Au NCs, whereas white blood cells (WBC), hematocrit, and HGB decreased significantly after 30 days. Besides, mean corpuscular volume (MCV) also decreased dramatically on the 60th day. In contrast, negative Au NCs only led to a decrease in WBC on day 90 and an increase in PLT on day 1. Neutral Au NCs caused an increase in PLT and MCHC and a decrease in MCV after 30 days. The results indicated that the surface charge has a huge influence on the toxicity of $\mathrm{Au}$ NCs, with the positive ones being the most prominent. This is consistent with previous in vitro studies and showed that positively charged NCs were more easily transported into cells,
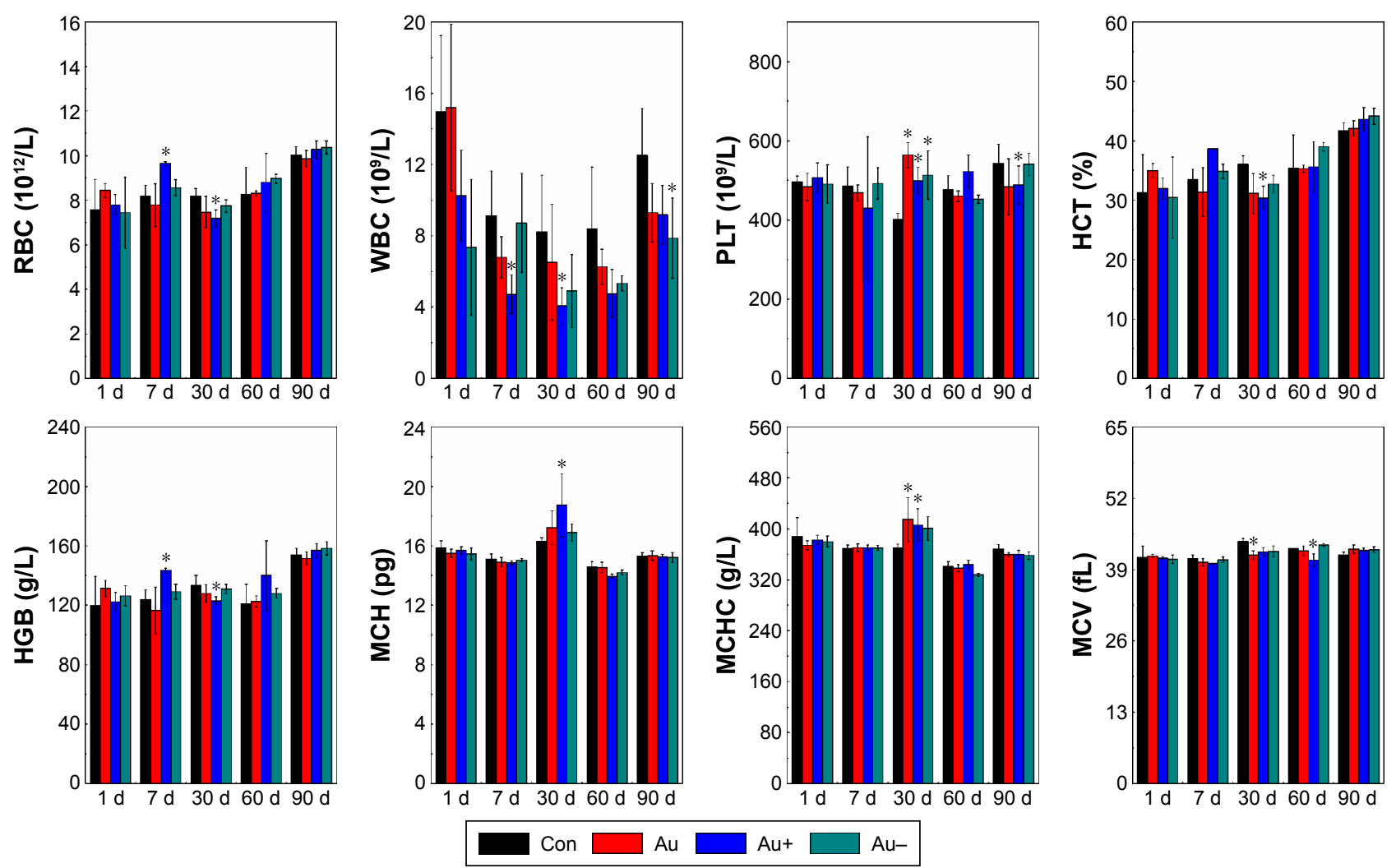

Figure 4 Hematology of WBC, RBC, PLT, HCT, HGB, MCH, MCHC, and MCV at I, 7, 30, 60, and 90 days postinjection.

Notes: *Indicates a significant difference between control and Au NC-treated groups, $P<0.05$. The $P$-value in each graph from left to right: RBC, $P=0.009$; WBC, $P=0.024$, 0.026, 0.035; PLT, $P=0.001,0.017,0.036 ; \mathrm{HCT}, P=0.017 ; \mathrm{HGB}, P=0.01,0.038 ; \mathrm{MCH}, P=0.039 ; \mathrm{MCHC}, P=0.049,0.026 ; \mathrm{MCV}, P=0.007,0.016$.

Abbreviations: Con, control; WBC, white blood cells; RBC, red blood cell; PLT, platelets; HCT, hematocrit; HGB, hemoglobin; MCH, mean corpuscular hemoglobin; $\mathrm{MCHC}$, mean corpuscular hemoglobin concentration; MCV, mean corpuscular volume; Au NCs, gold nanoclusters; d, days. 

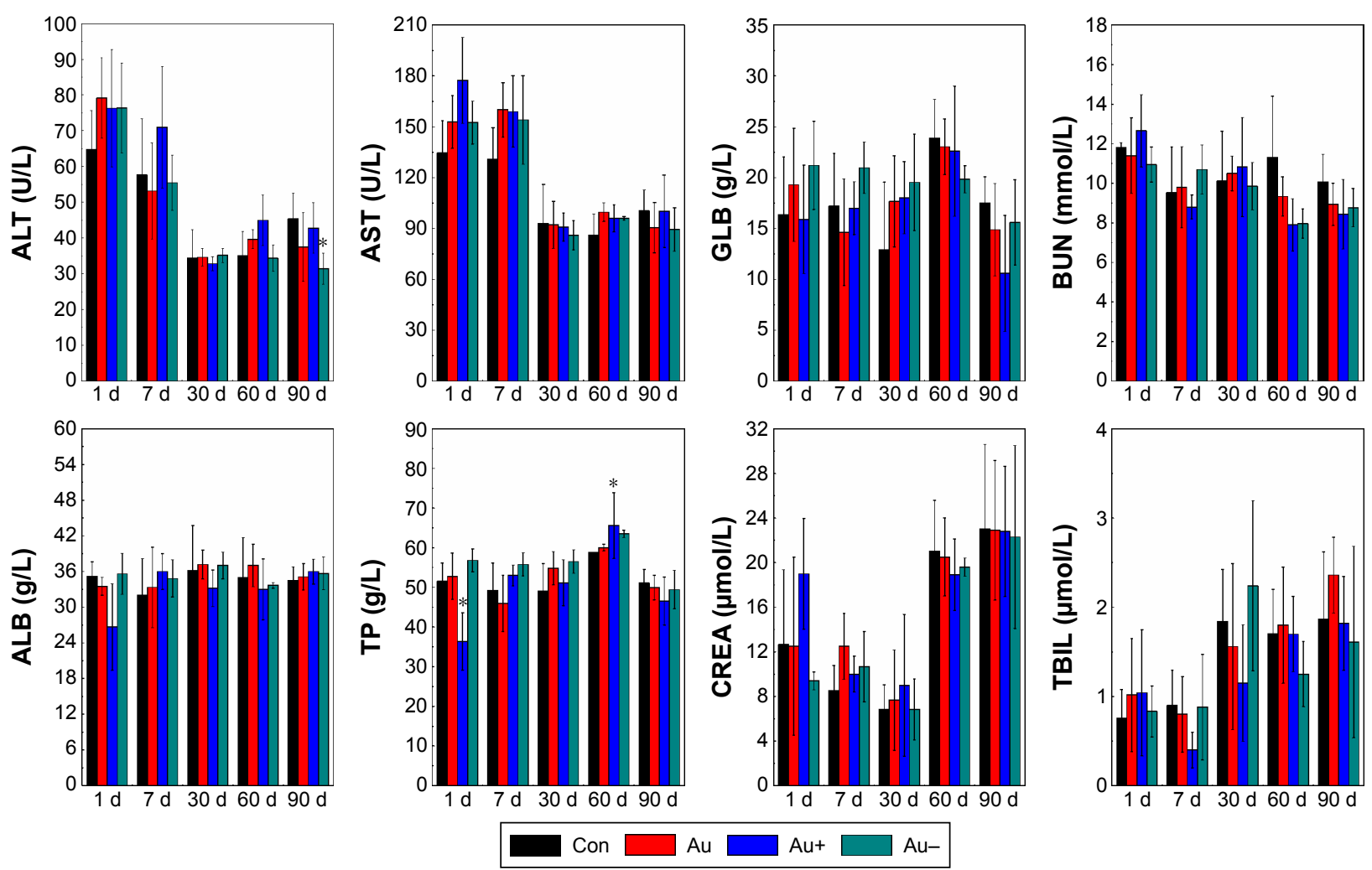

Figure 5 Biochemical levels of ALT, AST, GLB, BUN, ALB, TP, CREA, and TBIL at I, 7, 30, 60, and 90 days postinjection.

Notes: *Indicates a significant difference between control and Au NC-treated groups, $P<0.05$. The $P$-value in each graph from left to right: $A L T, P=0.033 ;$; $, P=0.033,0.049$. Abbreviations: ALT, alanine aminotransferase; AST, aspartate aminotransferase; GLB, globulin; BUN, blood urea nitrogen; ALB, albumin; TP, total protein; CREA, creatinine; TBIL, total bilirubin; Au NCs, gold nanoclusters; d, days.

resulting in the breakage of cell membranes. ${ }^{33}$ Importantly, all parameters recovered to normal levels after 90 days.

Biochemistry analysis was performed because the levels of particular enzymes and proteins in serum can indicate damage to important organs such as liver and kidney, which are targets for Au NCs. We measured alanine transferase (ALT), aspartate transferase, total protein (TP), albumin, globulin, and total bilirubin to evaluate the liver function after treatment with Au NCs and creatinine and blood urea nitrogen to evaluate the kidney function. As shown in Figure 5, ALT decreased significantly on day 90 for mice treated with negative $\mathrm{Au}$ $\mathrm{NCs}$, while positive $\mathrm{Au}$ NCs caused a dramatic increase in TP on days 1 and 90. Fortunately, none of the Au NCs showed any adverse effects on organ function, regardless of the surface charge. In view of the toxicity results described, which showed that kidney is the main organ that is targeted, we chose this organ for immunohistochemical analysis, and consistent with the biochemical analysis, no evident damage was apparent within 90 days of treatment (Figure 6).

\section{Therapy using Au NCs}

The surface charge of NPs has a great influence on the circulation time in blood and tumor uptake efficiency. ${ }^{48,52-56}$
$\mathrm{Au}$ NCs with different surface charges are promising candidates for tumor targeting during radiotherapy sensitization. We therefore studied the accumulation of Au NCs with different surface charges in tumor tissue using U14-bearing nude mice administered with $0.2 \mathrm{~mL}$ of $3 \mathrm{mM}$ Au NCs. At 24 hours postinjection, the $\mathrm{Au}$ concentration in tumor tissue was $149,222.6$, and $320.7 \mathrm{ng} / \mathrm{g}$ for neutral, positive, and negative NCs, respectively (Figure 7A). Negatively charged $\mathrm{Au}$ NCs exhibited the highest tumor uptake, and positive NCs also showed higher uptake compared to neutral clusters. The effects of surface charge on radiotherapy sensitization were further investigated using U14 tumor-bearing nude mice exposed to $5 \mathrm{~Gy}$ gamma radiations. The time-dependent volume of tumors was measured every other day, and both negatively and positively charged $\mathrm{Au} \mathrm{NCs}$ were better at inhibiting the tumor growth, due to a more efficient uptake than neutral Au NCs (Figure 7B). The average tumor volumes were $217 \mathrm{~mm}^{3}$ for radiation alone (control) and 175, 165, and $130 \mathrm{~mm}^{3}$ for groups treated with neutral, positive, and negative Au NCs, respectively. This corresponded to a tumor volume shrinkage of $38.9 \%, 42.2 \%$, and $54.3 \%$ after 23 days for neutral, positive, and negative Au NCs, respectively, compared with controls. $\mathrm{Au}$ NCs $(<5 \mathrm{~nm})$ are known to be superior to many other 

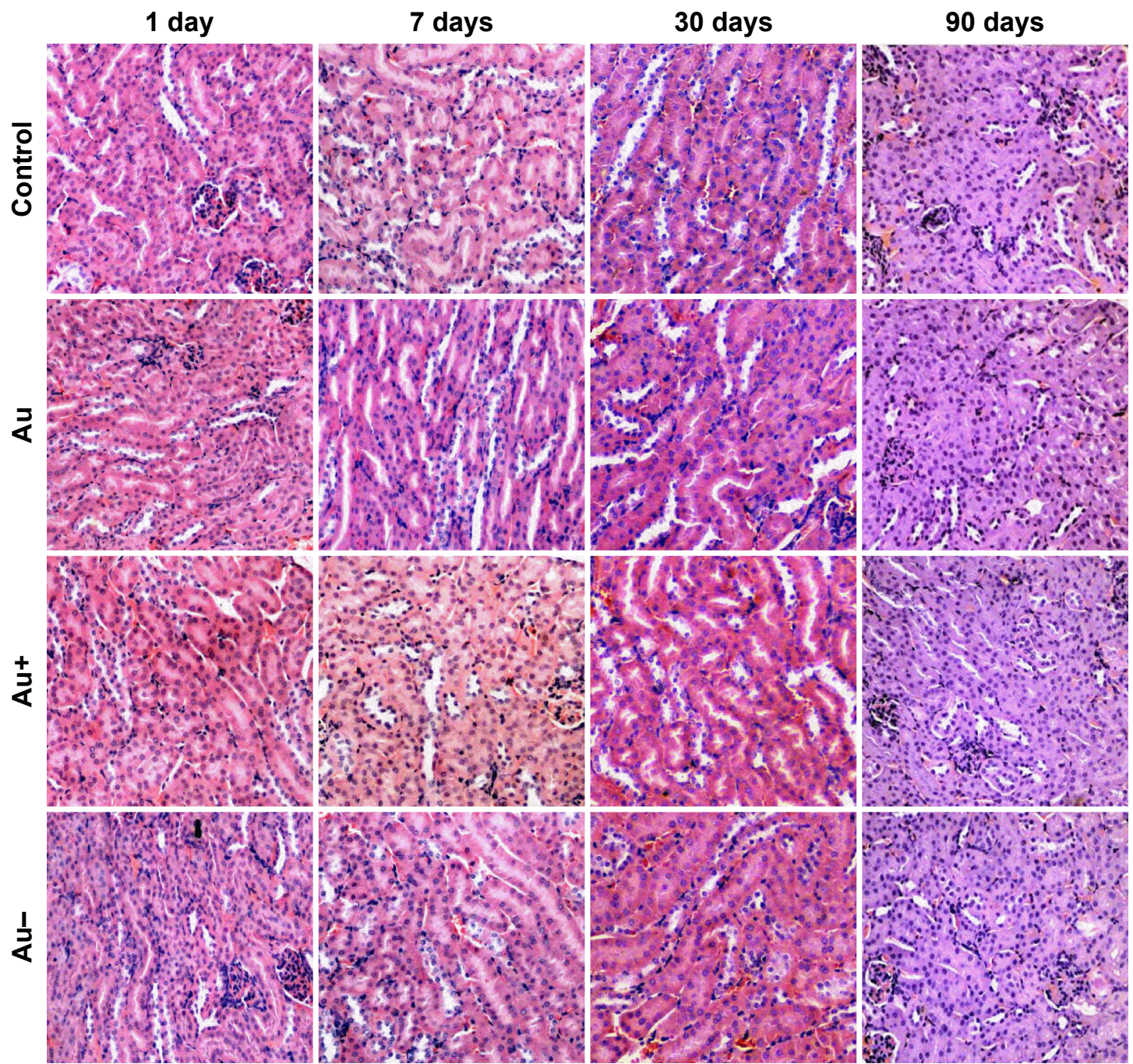

$100 \mu \mathrm{m}$

Figure 6 Kidney pathology following treatment with $\mathrm{Au}, \mathrm{Au}+$, and $\mathrm{Au}-$ at I, 7, 30, and 90 days postinjection. Abbreviation: Au, gold.

A Au NCs uptake in tumor 24 hours pi

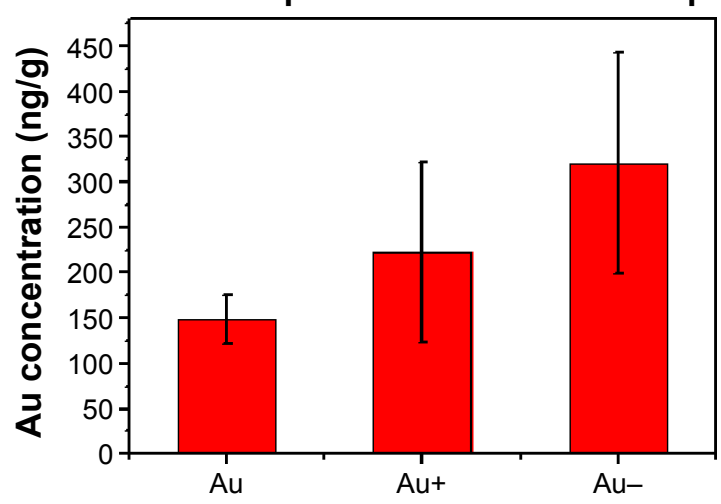

B

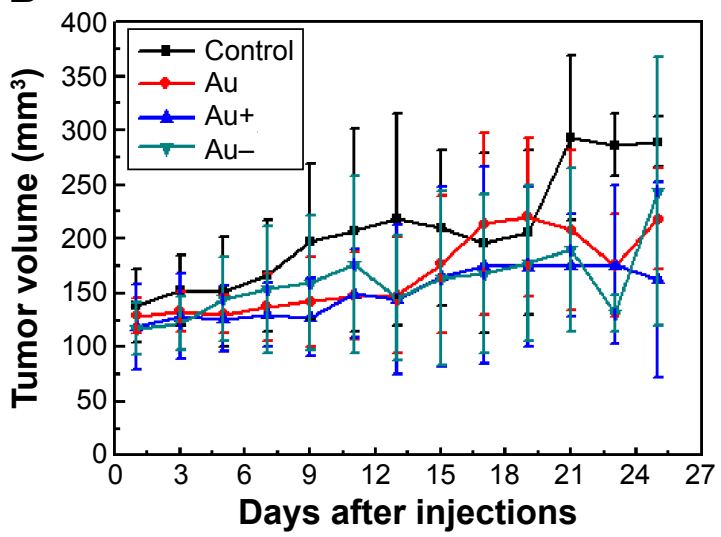

Figure 7 Tumor uptake and cancer therapy with $\mathrm{Au}, \mathrm{Au}+$, and $\mathrm{Au}$

Notes: (A) Biodistribution in tumor tissue of Au NCs with different surface charges at 24 hours after injection. (B) Change in tumor volume over 23 days following treatment with Au NCs under 5 Gy radiation.

Abbreviations: Au NCs, gold nanoclusters; pi, postinjection. 
particles in terms of tumor uptake and localization, and neutral $\mathrm{Au}$ NCs accumulate in tumor tissue due to enhanced permeability and retention as well as endothelial cell leakiness. ${ }^{51}$ However, fast renal clearance can undermine the potential for distribution in the tumor tissue. The high tumor uptake of positive $\mathrm{Au}$ NCs has been attributed to rapid extrication into the interstitium and internalization into the endothelium. ${ }^{57,58}$ Additionally, the cellular toxicity of positive Au NCs also contributed to enhancing the radiotherapeutic effects above those exhibited by neutral NCs. ${ }^{32,59}$ Negatively charged NCs exhibited the strongest antitumor effect mainly due to reduced nonspecific cellular uptake by the phagocytic system and adsorption by plasma proteins. ${ }^{54}$ Moreover, negative Au NCs with small size $(\sim 3.2 \mathrm{~nm})$ could be sheltered from the RES arrest more efficiently. He et al compared the effects of polymeric particles of different size and surface charges on tumor uptake and in vivo biodistribution, showing that rhodamine $B$ labeled carboxymethyl chitosan grafted nanoparticles (RhBCMCNP) bearing less negative charges exhibited enhanced tumor distribution and in correspondence with lowest liver and spleen containing. ${ }^{48}$ Therefore, longer circulation and stability in bloodstream could increase the potential for tumor uptake and distribution. ${ }^{54}$ In the present study, both positively and negatively charged Au NCs exhibited higher tumor uptake than neutral clusters. Negative Au NCs retained in vivo with prolonged circulations, which may explain the high tumor uptake. These results may be useful for designing Au NCs with more specific radiotherapy sensitization effects.

Au NCs of ultrasmall sizes can be highly active, and the surface charge plays an important role in determining both biodistribution and toxicology. ${ }^{60,61}$ In this study, we prepared three differently charged Au NCs (neutral, positive, and negative) and studied their stabilities in blood plasma, biodistribution, excretion, toxicity, and therapeutic potential. Compared with neutral Au NCs, negatively charged Au NCs were retained longer in liver and spleen, presumably due to capture by Kupffer cells and macrophages. ${ }^{43,52}$ Positive $\mathrm{Au}$ NCs caused some recoverable damage to the peripheral blood system, but normal functioning was recovered within the 90-day trial. Negative Au NCs showed the highest tumor uptake and the strongest effect in radiotherapy sensitization. Surface charge also played a critical role in the biocompatibility and therapeutic effects with charged clusters displaying better uptake efficiency that could benefit future therapies.

\section{Conclusion}

In this work, we prepared three differently charged Au NCs (neutral, positive, and negative) and studied their stability in blood plasma, biodistribution, excretion, toxicity, and therapeutic potential. Compared with neutral Au NCs, negatively charged Au NCs were retained longer in liver and spleen, presumably due to internalization by Kupffer cells and macrophages. Positive Au NCs caused a small amount of damage to the peripheral blood system, but normal function was recovered within 90 days postinjection. Surface charge had a pronounced effect on tumor uptake. Negative Au NCs displayed the highest tumor uptake and the most pronounced radiotherapy sensitization. Surface charge, therefore, plays an important role in toxicity and therapeutic potential for $\mathrm{Au} \mathrm{NCs.}$

\section{Acknowledgments}

This work was supported by the National Natural Science Foundation of China (Nos 81471786, 11304220, 81000668), Natural Science Foundation of Tianjin (Nos 13JCQNJC13500, 14JCYBJC26700), and the Subject Development Foundation of Institute of Radiation Medicine, CAMS (Nos SF1601, SF1503).

\section{Disclosure}

The authors report no conflicts of interest in this work.

\section{References}

1. Zhang XD, Chen J, Luo Z, et al. Enhanced tumor accumulation of sub-2 $\mathrm{nm}$ gold nanoclusters for cancer radiation therapy. Adv Healthcare Mater. 2014;3(1):133-141.

2. Zhang XD, Luo Z, Chen J, et al. Ultrasmall $\mathrm{Au}_{10-12}(\mathrm{SG})_{10-12}$ nanomolecules for high tumor specificity and cancer radiotherapy. Adv Mater. 2014; 26(26):4565-4568.

3. Liu J, Yu M, Zhou C, Yang S, Ning X, Zheng J. Passive tumor targeting of renal-clearable luminescent gold nanoparticles: long tumor retention and fast normal tissue clearance. J Am Chem Soc. 2013;135(13):4978-4981.

4. Zhang XD, Wu D, Shen X, et al. Size-dependent in vivo toxicity of PEG-coated gold nanoparticles. Int J Nanomed. 2011;6:2071-2081.

5. Chen J, Wang H, Long W, et al. Sex differences in the toxicity of polyethylene glycol-coated gold nanoparticles in mice. Int J Nanomed. 2013;8:2409-2419.

6. Fernandes R, Smyth NR, Muskens OL, et al. Interactions of skin with gold nanoparticles of different surface charge, shape, and functionality. Small. 2015;11(6):713-721.

7. Choi HS, Liu W, Misra P, et al. Renal clearance of quantum dots. Nat Biotechnol. 2007;25(10):1165-1170.

8. Zheng J, Zhou C, Yu M, Liu J. Different sized luminescent gold nanoparticles. Nanoscale. 2012;4(14):4073-4083.

9. Zhang XD, Chen J, Yang J, et al. Use of epidermal growth factor receptor antibody-gold cluster conjugates with good renal excretion in targeted cancer radiation treatment. J Mater Chem B. 2015;3(23):4735-4741.

10. Yang Y, Gao N, Hu Y, et al. Gold nanoparticle-enhanced photodynamic therapy: effects of surface charge and mitochondrial targeting. Ther Deliv. 2015;6(3):307-321.

11. Retnakumari A, Setua S, Menon D, et al. Molecular-receptor-specific, non-toxic, near-infrared-emitting $\mathrm{Au}$ cluster-protein nanoconjugates for targeted cancer imaging. Nanotechnology. 2010;21(5):055103.

12. Zhang X, Wu FG, Liu P, Wang HY, Gu N, Chen Z. Synthesis of ultrastable and multifunctional gold nanoclusters with enhanced fluorescence and potential anticancer drug delivery application. J Colloid Interface Sci. 2015;455:6-15. 
13. Zhang XD, Wu D, Shen $X$, et al. Size-dependent radiosensitization of PEG-coated gold nanoparticles for cancer radiation therapy. Biomaterials. 2012;33(27):6408-6419.

14. Zhang XD, Luo Z, Chen J, et al. Storage of gold nanoclusters in muscle leads to their biphasic in vivo clearance. Small. 2015;11(14): 1683-1690.

15. Zhou C, Long M, Qin Y, Sun X, Zheng J. Luminescent gold nanoparticles with efficient renal clearance. Angew Chem Int Ed. 2011; 50(14):3168-3172.

16. Shang L, Dong S, Nienhaus GU. Ultra-small fluorescent metal nanoclusters: synthesis and biological applications. Nano Today. 2011;6(4): 401-418.

17. Lin CA, Yang TY, Lee CH, et al. Synthesis, characterization, and bioconjugation of fluorescent gold nanoclusters toward biological labeling applications. ACS Nano. 2009;3(2):395-401.

18. Lin Y, Wu Z, Wen J, et al. Adhesion and atomic structures of gold on ceria nanostructures: the role of surface structure and oxidation state of ceria supports. Nano Lett. 2015;15(8):5375-5381.

19. Chithrani BD, Chan WC. Elucidating the mechanism of cellular uptake and removal of protein-coated gold nanoparticles of different sizes and shapes. Nano Lett. 2007;7(6):1542-1550.

20. Albanese A, Tang PS, Chan WC. The effect of nanoparticle size, shape, and surface chemistry on biological systems. Annu Rev Biomed Eng. 2012;14:1-16.

21. Walkey CD, Olsen JB, Guo H, Emili A, Chan WC. Nanoparticle size and surface chemistry determine serum protein adsorption and macrophage uptake. J Am Chem Soc. 2012;134(4):2139-2147.

22. Bozich JS, Lohse SE, Torelli MD, Murphy CJ, Hamers RJ, Klaper RD. Surface chemistry, charge and ligand type impact the toxicity of gold nanoparticles to Daphnia magna. Environ Sci Nano. 2014;1(3):260-270.

23. Zhang XD, Wu HY, Wu D, et al. Toxicologic effects of gold nanoparticles in vivo by different administration routes. Int J Nanomed. 2010; 5:771-781.

24. Connor EE, Mwamuka J, Gole A, Murphy CJ, Wyatt MD. Gold nanoparticles are taken up by human cells but do not cause acute cytotoxicity. Small. 2005;1(3):325-327.

25. Shukla R, Bansal V, Chaudhary M, Basu A, Bhonde RR, Sastry M. Biocompatibility of gold nanoparticles and their endocytotic fate inside the cellular compartment: a microscopic overview. Langmuir. 2005;21(23): 10644-10654.

26. Li JJ, Hartono D, Ong CN, Bay BH, Yung LY. Autophagy and oxidative stress associated with gold nanoparticles. Biomaterials. 2010;31(23): 5996-6003.

27. Ng CT, Li JJ, Gurung RL, et al. Toxicological profile of small airway epithelial cells exposed to gold nanoparticles. Exp Biol Med. 2013;238(12): 1355-1361.

28. Li L, Zhang L, Wang T, et al. Facile and scalable synthesis of novel spherical Au nanocluster assemblies@polyacrylic acid/calcium phosphate nanoparticles for dual-modal imaging-guided cancer chemotherapy. Small. 2015;11(26):3162-3173.

29. Chen H, Li S, Li B, et al. Folate-modified gold nanoclusters as nearinfrared fluorescent probes for tumor imaging and therapy. Nanoscale. 2012;4(19):6050-6064.

30. Wang $\mathrm{HH}$, Lin CA, Lee $\mathrm{CH}$, et al. Fluorescent gold nanoclusters as a biocompatible marker for in vitro and in vivo tracking of endothelial cells. ACS Nano. 2011;5(6):4337-4344.

31. Jenkins JT, Halaney DL, Sokolov KV, et al. Excretion and toxicity of gold-iron nanoparticles. Nanomedicine. 2013;9(3):356-365.

32. Schaeublin NM, Braydich Stolle LK, Schrand AM, et al. Surface charge of gold nanoparticles mediates mechanism of toxicity. Nanoscale. 2011;3(2):410-420.

33. Yue ZG, Wei W, Lv PP, et al. Surface charge affects cellular uptake and intracellular trafficking of chitosan-based nanoparticles. Biomacromolecules. 2011;12(7):2440-2446.

34. Asati A, Santra S, Kaittanis C, Perez JM. Surface-charge-dependent cell localization and cytotoxicity of cerium oxide nanoparticles. ACS Nano. 2010;4(9):5321-5331.
35. Shang L, Yang L, Seiter J, et al. Nanoparticles interacting with proteins and cells: a systematic study of protein surface charge effects. Adv Mater Interfaces. 2014;1(2).

36. Calatayud MP, Sanz B, Raffa V, Riggio C, Ibarra MR, Goya GF. The effect of surface charge of functionalized $\mathrm{Fe}_{3} \mathrm{O}_{4}$ nanoparticles on protein adsorption and cell uptake. Biomaterials. 2014;35(24):6389-6399.

37. Su GX, Zhou HY, Mu QX, et al. Effective surface charge density determines the electrostatic attraction between nanoparticles and cells. J Phys Chem C. 2012;116(8):4993-4998.

38. Ghosh PS, Kim CK, Han G, Forbes NS, Rotello VM. Efficient gene delivery vectors by tuning the surface charge density of amino acid-functionalized gold nanoparticles. ACS Nano. 2008;2(11):2213-2218.

39. El Badawy AM, Silva RG, Morris B, Scheckel KG, Suidan MT, Tolaymat TM. Surface charge-dependent toxicity of silver nanoparticles. Environ Sci Technol. 2011;45(1):283-287.

40. Souris JS, Lee $\mathrm{CH}$, Cheng $\mathrm{SH}$, et al. Surface charge-mediated rapid hepatobiliary excretion of mesoporous silica nanoparticles. Biomaterials. 2010;31(21):5564-5574.

41. Yuan YY, Mao CQ, Du XJ, Du JZ, Wang F, Wang J. Surface charge switchable nanoparticles based on zwitterionic polymer for enhanced drug delivery to tumor. Adv Mater. 2012;24(40):5476-5480.

42. Frohlich $\mathrm{E}$. The role of surface charge in cellular uptake and cytotoxicity of medical nanoparticles. Int J Nanomed. 2012;7:5577-5591.

43. Luo Z, Yuan X, Yu Y, et al. From aggregation-induced emission of $\mathrm{Au}(\mathrm{I})$-thiolate complexes to ultrabright $\mathrm{Au}(0) @ \mathrm{Au}(\mathrm{I})$-thiolate core-shell nanoclusters. J Am Chem Soc. 2012;134(40):16662-16670.

44. Gessner A, Lieske A, Paulke BR, Muller RH. Functional groups on polystyrene model nanoparticles: influence on protein adsorption. J Biomed Mater Res A. 2003;65(3):319-326.

45. Aggarwal P, Hall JB, McLeland CB, Dobrovolskaia MA, McNeil SE. Nanoparticle interaction with plasma proteins as it relates to particle biodistribution, biocompatibility and therapeutic efficacy. Adv Drug Deliv Rev. 2009;61(6):428-437.

46. Barran-Berdon AL, Pozzi D, Caracciolo G, et al. Time evolution of nanoparticle-protein corona in human plasma: relevance for targeted drug delivery. Langmuir. 2013;29(21):6485-6494.

47. Balogh L, Nigavekar SS, Nair BM, et al. Significant effect of size on the in vivo biodistribution of gold composite nanodevices in mouse tumor models. Nanomedicine. 2007;3(4):281-296.

48. He CB, Hu YP, Yin LC, Tang C, Yin CH. Effects of particle size and surface charge on cellular uptake and biodistribution of polymeric nanoparticles. Biomaterials. 2010;31(13):3657-3666.

49. Zhang XD, Yang J, Song SS, et al. Passing through the renal clearance barrier: toward ultrasmall sizes with stable ligands for potential clinical applications. Int J Nanomed. 2014;9:2069-2072.

50. Zhang XD, Wu D, Shen X, Liu PX, Fan FY, Fan SJ. In vivo renal clearance, biodistribution, toxicity of gold nanoclusters. Biomaterials. 2012;33(18):4628-4638.

51. Setyawati MI, Tay CY, Chia SL, et al. Titanium dioxide nanomaterials cause endothelial cell leakiness by disrupting the homophilic interaction of VE-cadherin. Nat Commun. 2013;4:1673.

52. Hirn S, Semmler Behnke M, Schleh C, et al. Particle size-dependent and surface charge-dependent biodistribution of gold nanoparticles after intravenous administration. Eur J Pharm Biopharm. 2011;77(3): 407-416.

53. Xiao K, Li Y, Luo J, et al. The effect of surface charge on in vivo biodistribution of PEG-oligocholic acid based micellar nanoparticles. Biomaterials. 2011;32(13):3435-3446.

54. Alexis F, Pridgen E, Molnar LK, Farokhzad OC. Factors affecting the clearance and biodistribution of polymeric nanoparticles. Mol Pharm. 2008;5(4):505-515.

55. Nigavekar SS, Sung LY, Llanes M, et al. $3 \mathrm{H}$ dendrimer nanoparticle organ/tumor distribution. Pharm Res. 2004;21(3):476-483.

56. Yang K, Wan JW, Zhang S, Tian B, Zhang YJ, Liu Z. The influence of surface chemistry and size of nanoscale graphene oxide on photothermal therapy of cancer using ultra-low laser power. Biomaterials. 2012;33(7):2206-2214. 
57. Ishiwata H, Suzuki N, Ando S, Kikuchi H, Kitagawa T. Characteristics and biodistribution of cationic liposomes and their DNA complexes. J Control Release. 2000;69(1):139-148.

58. Fang J, Nakamura H, Maeda H. The EPR effect: unique features of tumor blood vessels for drug delivery, factors involved, and limitations and augmentation of the effect. Adv Drug Deliver Rev. 2011;63(3): $136-151$.

59. Goodman CM, McCusker CD, Yilmaz T, Rotello VM. Toxicity of gold nanoparticles functionalized with cationic and anionic side chains. Bioconjugate Chem. 2004;15(4):897-900.
60. Zhang E, Fu A. A new strategy for specific imaging of neural cells based on peptide-conjugated gold nanoclusters. Int J Nanomed. 2015;10: 2115-2124.

61. Swanner J, Mims J, Carroll DL, et al. Differential cytotoxic and radiosensitizing effects of silver nanoparticles on triple-negative breast cancer and non-triple-negative breast cells. Int J Nanomed. 2015;10: 3937-3953.
International Journal of Nanomedicine

\section{Publish your work in this journal}

The International Journal of Nanomedicine is an international, peerreviewed journal focusing on the application of nanotechnology in diagnostics, therapeutics, and drug delivery systems throughout the biomedical field. This journal is indexed on PubMed Central, MedLine, CAS, SciSearch ${ }^{\circledR}$, Current Contents ${ } /$ Clinical Medicine,

\section{Dovepress}

Journal Citation Reports/Science Edition, EMBase, Scopus and the Elsevier Bibliographic databases. The manuscript management system is completely online and includes a very quick and fair peer-review system, which is all easy to use. Visit http://www.dovepress.com/ testimonials.php to read real quotes from published authors.

Submit your manuscript here: http://www.dovepress.com/international-journal-of-nanomedicine-journal 